\title{
Therapeutic Leukapheresis: Experience of a Single Oncologic Centre
}

\author{
Maria Rosales $^{a}$ Susana Roncon $^{b}$ Mário Mariz ${ }^{c}$ Ana Maia Ferreira ${ }^{d}$ \\ Filomena Faria $^{\mathrm{e}}$ Luisa Santos ${ }^{\mathrm{a}}$ \\ ${ }^{a}$ Hemotherapy Service, Portuguese Oncology Institute of Porto (IPO Porto), Porto, Portugal; ${ }^{b}$ Cell Therapy Service, \\ Portuguese Oncology Institute of Porto (IPO Porto), Porto, Portugal; ' ${ }^{\circ}$ nco-Hematology Service, Portuguese

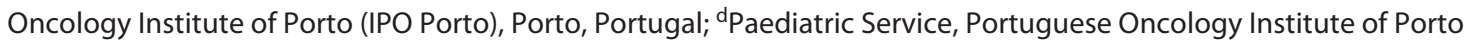 \\ (IPO Porto), Porto, Portugal; ' Intensive Care Unit, Portuguese Oncology Institute of Porto (IPO Porto), Porto, Portugal
}

\section{Keywords}

Therapeutic leukapheresis

\begin{abstract}
Introduction: Leukostasis refers to clinical symptoms caused by hyperleukocytosis seen in some haematological diseases such as leukaemia. Cytoreduction can be achieved by therapeutic leukapheresis. The aim of this study was to retrospectively analyse the procedures performed in our Centre and to evaluate their efficacy and safety. Methods: This was a retrospective study of all the therapeutic leukapheresis procedures carried out in our Centre between January 1998 and December 2020. The sample collection was obtained through the review of the clinical files of the respective patients. Statistical analysis was performed using the software R v.4.0.1. A total of 54 therapeutic leukapheresis procedures were performed in 31 patients in our Centre. Results: After these procedures clinical improvement was observed in 16 patients and we verify that there was a significant difference in survival between the group that improved and the group that maintained the same clinical condition or worsened. The lack of immediate clinical improvement was a sign of a poor prognosis. Laboratory efficacy occurred in 16 patients who had a reduction in white blood cell count, with a $39.1 \%$ reduction after $24 \mathrm{~h}$, and did not succeed in 15 patients, who had no reduction. However, in this case there is no significant difference in survival between the two groups. There was some complication in $53.9 \%$ of the procedures, with hypocalcaemia being the most frequent, which
\end{abstract}

\section{karger@karger.com} www.karger.com/tmh

Karger $\frac{1}{*}$

BOPEN ACCESS
(C) 2022 The Author(s)

Published by S. Karger AG, Basel

This is an Open Access article licensed under the Creative Common Attribution-NonCommercial-4.0 International License (CC BY-NC) (http://www.karger.com/Services/OpenAccessLicense), applicable to the online version of the article only. Usage and distribution for commercial purposes requires written permission. was observed in 22 procedures. Only 4 patients experienced serious side effects but these adverse reactions cannot be attributed to the procedures carried out. The overall survival rate 6 months after this treatment was $51.6 \%$. Conclusion: Despite the reduced number of patients, we conclude that therapeutic leukapheresis is a safe and effective option that may still have a therapeutic role in some cases.

() 2022 The Author(s).

Published by S. Karger AG, Basel

\section{Introduction}

Hyperleukocytosis (HL) is defined as a white blood cell (WBC) count greater than $100 \times 10^{9} / \mathrm{L}$, and is a complication seen in patients with some haematological neoplasms such as acute leukaemia. Risk factors include younger age, certain cytogenetic abnormalities, and monocytic differentiation subtypes [1]. The incidence of HL ranges between 5 and 13\% in adult acute myeloid leukaemia (AML) and between 10 and $30 \%$ in acute lymphoblastic leukaemia (ALL), and is often associated with high early rates of morbidity and mortality related to the possible development of leukostasis, tumour lysis syndrome (TLS), and/or disseminated intravascular coagulopathy (DIC) [2-4]. Leukostasis refers to clinical symptoms and complications caused by HL, but the clinical diagnosis is rarely made with high confidence because it is difficult to distinguish from those clinical and radiographic manifestations of common infections or haemorrhagic complications of acute leukaemia. The central nervous system and 
lungs are the most common places for symptomatic vascular obstruction, but effects on other organ systems can occur. Both neurological and pulmonary manifestations are associated with increased rates of mortality [5]. Cytoreduction can be achieved either by mechanical removal of excessive leukocytes in the peripheral blood via therapeutic leukapheresis (TL) or pharmacologic strategies, in order to prevent potential complications in patients presenting HL. The TL procedure refers to the withdrawal of the blood from the body, the separation and removal of WBC, and the subsequent infusion of the other constituents back into the patient. Due to an excessive early mortality, HL in leukaemia is a medical emergency that needs prompt recognition. Treatment should start as soon as clinical signs of leukostasis appear to avoid pulmonary, cerebral, and even cardiac leukostasis due to the excess WBC and to reduce the risk of tissue hypoxia, DIC, and TLS induced by the cellular lysis caused by chemotherapy [6]. Leukapheresis is an effective means of cytoreduction that has been used in these patients with the goal of reducing the number of WBC in the circulation. TL is used to treat or prevent the consequences due to hyperviscosity and may have an immediate effect resulting in rapid improvement in the patient's condition $[7,8]$. Contraindications such as cardiovascular comorbidities, hemodynamic instability, and coagulation disturbances should be evaluated carefully in order to avoid risks for the patient. TL has become routinely available in many hematologic treatment centres and so can be performed, but without delaying the supportive treatment with hydration, allopurinol or rasburicase, and hydroxyurea [9]. Actually, TL is being used less and less compared with the end of the last century because other treatments, such as supportive treatment and chemotherapy, have made enormous progress. However, in some patients in a poor condition, TL remains an option. In modern apheresis devices, WBC and their precursors are separated from the patients' blood by centrifugation and during a single TL procedure the WBC count can be reduced by $10-70 \%$. Although the clinical and analytical efficacy of TL to reduce the number of WBCs has been proven in several clinical trials, its use in HL patients is still under debate. It happens that most of the leukemic burden is located in the bone marrow and these cells are rapidly mobilized into the peripheral blood shortly after a successful TL and a beneficial clinical effect on early outcome could not be shown consistently in clinical trials. Published data of the retrospective studies regarding the clinical value of TL for cytoreduction is not well documented in the literature and is still unclear. There are limited, observational, and conflicting results, and more evidence is needed considering the lack of randomised prospective controlled trials, so interpretation of these studies should be made carefully [10]. The ideal therapeutic approach in the absence of these randomised clinical trials remains controversial. The British TL guidelines state that it can be used as part of the management of HL when it is complicated by clinical leukostasis or when the use of chemotherapy is problematic as it occurs during pregnancy, with a strong recommendation but low-quality evidence [11]. The American TL guidelines have a weak recommendation with moderate-quality evidence for the symptomatic HL, while they have a weak recommendation, with low-quality or very low-quality evidence for prophylactic or secondary HL, and decision making should be individualised [12]. TL did not lead to an improvement in the primary outcome of early mortality compared to treatment strategies that did not employ leukapheresis. Response to treatment is monitored by its effects on clinical symptoms and/or reduction of cell count by at least $20 \%$. For patients with AML and leukostasis complications, apheresis must be discontinued when the blast cell count is less than $50-100 \times 10^{9} / \mathrm{L}$ and clinical manifestations are resolved or maximum benefit is achieved. Chemotherapy should not be postponed and is required to prevent the rapid accumulation of circulating blasts. TL generally is performed in acutely ill patients with severe thrombocytopenia and a coagulopathy due to acute leukaemia. In these cases, the placement of a catheter may be difficult, but it is recommended that a dialysis-compatible central venous catheter is placed not to compromise the efficacy of the procedure in unstable patients that may need more than one procedure. Although TL is performed safely in most cases, adverse reactions can occasionally be seen, and their pattern and frequency of occurrence should be well known to the practicing haematologist. The most frequent problem is related to the anticoagulant used to prevent clotting of the apheresis circuit. Citrate binds calcium and induces symptomatic hypocalcaemia with paraesthesia and muscle cramps as a result from the fact that the citrate anticoagulant of the blood products binds to the free calcium in the patient's plasma to form soluble calcium citrate, leading to low free calcium levels. Other potential side effects are nausea, vomiting, dizziness, seizures, skin rash, hives, and flushing. There are also risks related to venous access, so irritation, bruising, swelling, hematomas, and infection are potential risks. More serious complications are less common [13]. Blood loss is a known side effect because contaminating red blood cells $(\mathrm{RBC})$ are collected with $\mathrm{WBC}$. By reducing the $\mathrm{WBC}$, platelets and $\mathrm{RBC}$ also have varying degrees of reduction simultaneously. Among these decreased blood components monocytes, neutrophils, and platelets, decreased the most, suggesting that potential haemorrhage and infection should be taken into consideration. Some centres prefer more conventional treatment in asymptomatic and paediatric patients due to the invasive nature of this technique, the need for experienced staff, and the central venous access as well as the 
additional costs and limited evidence proving its efficacy [14]. Few larger reports on leukapheresis safety exist but, in the hands of apheresis specialists, it is apparently quite safe for patients and its adverse effects are mostly manageable [15].

\section{Aims}

The aim of this study was to retrospectively analyse the TL procedures performed in our centre between January 1998 and December 2020, and also to evaluate its efficacy and safety.

\section{Methods}

This was a retrospective study of all the TL procedures carried out in our centre between January 1998 and December 2020. The sample collection was obtained from the records of the Hemotherapy Service through the review of the clinical files of the respective patients. The information of TL sessions was registered at the end of each of them and we analysed the procedures and the results by consulting the clinical records.

\section{Statistical Analysis}

Continuous variables are presented as the median (minimum and maximum), and categorical variables are presented as frequencies and percentages. Overall survival and its corresponding $95 \%$ confidence intervals (CI) were estimated by the Kaplan-Meier method. The log-rank test was performed to compare survival rates between groups. Statistical significance was considered at the level of $p<0.05$. The statistical analysis was performed using the software R v.4.0.1.

\section{Results}

Our hospital is the largest cancer care institution in Portugal and during this period many leukaemia patients were admitted to the Onco-Hematology and Paediatric Services. Whenever a patient with leukostatic symptoms was diagnosed with an exuberant clinic and without contraindication for the procedure, TL was chosen as part of the treatment. A total of $54 \mathrm{TL}$ were performed in $31 \mathrm{pa}-$ tients. Most patients were female (16, 51.6\%; Table 1$)$, and the median age was 22 years (2-77). Sixteen (51.6\%) patients were diagnosed with AML, 11 (35.5\%) with ALL, and $4(12.9 \%)$ with chronic myeloid leukaemia (CML). All patients initiated TL within 1 week after the diagnosis and it was necessary for patients to enter the intensive care unit. Note that from 2015 the admission policy to the intensive care unit changed and paediatric patients who needed intensive care were transferred to another hospital. Only 5 patients $(16.1 \%)$ did not have leukostatic symptoms and the TL decision was based on the high number of peripheral WBC in the rational of early initiation of suitable chemotherapy and good supportive care when it was considered to achieve rapid cytoreduction as
Table 1. Patient characteristics and therapeutic leukapheresis efficacy $(n=31)$

\begin{tabular}{|c|c|}
\hline Age, years & $22(2-77)$ \\
\hline Weight, kg & $63(13-112)$ \\
\hline Volemia, mL & $3,928(838-5,849)$ \\
\hline \multicolumn{2}{|l|}{ Sex } \\
\hline Male & $15(48.4)$ \\
\hline Female & $16(51.6)$ \\
\hline \multicolumn{2}{|l|}{ Diagnosis } \\
\hline AML & $16(51.6)$ \\
\hline AML-M1 & $3(9.7)$ \\
\hline AML-M2 & $1(3.2)$ \\
\hline AML-M3 & $2(6.5)$ \\
\hline AML-M4 & $5(16.1)$ \\
\hline AML-M5 & $5(16.1)$ \\
\hline ALL & $11(35.5)$ \\
\hline ALL-B & $3(9.7)$ \\
\hline ALL-T & $8(25.8)$ \\
\hline CML & $4(12.9)$ \\
\hline \multicolumn{2}{|l|}{$\mathrm{TL}$} \\
\hline 1 session & $14(45.2)$ \\
\hline 2 sessions & $13(41.9)$ \\
\hline 3 sessions & $2(6.5)$ \\
\hline 4 sessions & $2(6.5)$ \\
\hline \multicolumn{2}{|l|}{ Leukostasis } \\
\hline Neurological & $5(16.1)$ \\
\hline Pulmonary & $9(29)$ \\
\hline Neurological and pulmonary & $12(38.7)$ \\
\hline No leukostasis & $5(16.1)$ \\
\hline \multicolumn{2}{|l|}{ Clinical efficacy } \\
\hline Improvement & $16(51.6)$ \\
\hline Same state or worse & $15(48.4)$ \\
\hline \multicolumn{2}{|l|}{ Laboratorial efficacy } \\
\hline WBC count reduction & $16(51.6)$ \\
\hline No WBC count reduction & $15(48.4)$ \\
\hline \multicolumn{2}{|l|}{ Status } \\
\hline Alive & $11(35.5)$ \\
\hline Dead & $20(64.5)$ \\
\hline \multicolumn{2}{|l|}{ Cause of death } \\
\hline ARDS & $6(30.0)$ \\
\hline Sepsis & $2(10.0)$ \\
\hline Sepsis + ARDS & $2(10.0)$ \\
\hline Sepsis + coagulopathy & $1(5.0)$ \\
\hline Sepsis + multiorgan failure & $2(10.0)$ \\
\hline Cardiorespiratory failure & $1(5.0)$ \\
\hline Cerebral haemorrhage & $2(10.0)$ \\
\hline Progression of underlying disease & $4(20.0)$ \\
\hline
\end{tabular}

Age (years), weight $(\mathrm{kg})$, and volemia $(\mathrm{mL})$ are presented as the median (minimum and maximum). Other values are presented as $n(\%)$.

a prophylactic treatment for TLS. The other patients presented cerebral manifestations $(5,16.1 \%)$, such as lethargy, aphasia, dysarthria, altered vision, and intracranial haemorrhage, and pulmonary manifestations (9, 29.0\%) that can include dry cough, respiratory distress, and alveolar haemorrhage or both simultaneously $(12,38.7 \%)$. The initial LDH level in serum was >1,000 U/L: 1,518 U/L $(478-8,002)$. All patients required a central venous cath- 
Table 2. TL procedure and product obtained $(n=54)$

\begin{tabular}{lll}
\hline Procedure & Blood volumes processed, $n$ & $2.0(0.8-4.0)$ \\
& Duration of the procedure, $\min$ & $118.5(22.0-283.0)$ \\
Product & Anticoagulant used, $\mathrm{mL}$ & $452(50-1,043)$ \\
Peripheral blood & Volume of product obtained, $\mathrm{mL}$ & $215.5(16-2,725)$ \\
& Leukocytes $/ \mathrm{mm}^{3}$ (before $\left.\mathrm{TL}\right)$ & $241,490(61,100-856,000)$ \\
Cellular separator & Leukocytes $/ \mathrm{mm}^{3}$ (after $\left.\mathrm{TL} ; n=53\right)$ & $180,570(10,900-514,560)$ \\
& \% leukocyte reduction $(n=53)$ & $39.1(-60.8$ to 88.9$)$ \\
& Cobe Spectra & $46(85.2)$ \\
\hline
\end{tabular}

Data are presented as the median (minimum and maximum) or $n$ (\%).

Table 3. TL complications and transfusion requirements $(n=54)$

\begin{tabular}{ll}
\hline Complications & \\
\hline Paresthesias & $22(40.7)$ \\
Respiratory arrest/failure & $2(3.7)$ \\
Arterial hypotension & $1(1.9)$ \\
Tachycardia & $1(1.9)$ \\
Bleeding & $1(1.9)$ \\
Apheresis kit obstruction & $1(1.9)$ \\
Hyperthermia & $1(1.9)$ \\
Transfusion requirements & \\
Tubing set priming & $15(27.8)$ \\
Erythrocyte concentrate units & $21(38.9)$ \\
Platelet pool/apheresis platelet concentrate units & $24(44.4)$ \\
Fresh frozen plasma & $16(29.6)$ \\
\hline
\end{tabular}

Data are presented as $n(\%)$.

eter for the procedure. Aphaeresis was performed using the continuous flow device Cobe Spectra cell separator (Terumo BCT) with the polymorphonuclear cells or mononuclear cell programs (MNC). Generally, the first one was the protocol selected for AML and the second for the rest. Spectra Optia cell separator (Terumo BCT) was used from 2015 with the protocol for WBC depletion, continuous $\mathrm{MNC}$ regardless of underlying disease. A median of 2 blood volumes per TL were processed (0.8-4.0; Table 2), this was normally programmed initially and adapted according to the evolution of the procedure. The median duration of the procedure was $118.5 \mathrm{~min}(22.0-$ 283.0) and each patient was treated with a median of $1 \mathrm{TL}$ session (1-4). The median pre-aphaeresis WBC count was $241.49 \times 10^{9} / \mathrm{L}(61.10-856.00)$ and the median WBC count $24 \mathrm{~h}$ after TL was $180.57 \times 10^{9} / \mathrm{L}(10.90-514.56)$. An efficacy index (EI) was calculated to monitor the procedures: $\mathrm{EI}=$ (total pre-aphaeresis patient $\mathrm{WBC}-$ total post-aphaeresis patient $\mathrm{WBC}$ )/total pre-aphaeresis patient $\mathrm{WBC} \times 100$, as the percentage of WBC reduction in this series. The median EI of all TL $(n=53$, because a patient died on the day of the last session and there was no blood test after $24 \mathrm{~h}$ ) was $39.1 \%$ (-60.8 to 88.9 ) and if we eliminate the TL sessions in which there was a rise in the WBC value after $24 \mathrm{~h}$, the EI $(n=45)$ would be $43.2 \%$ $(9.7-88.9)$. The anticoagulant used was an acid-citratedextrose solution A (ACD-A), the amount used was 452 $\mathrm{mL}(50-1,043)$, and the blood/anticoagulant ratio ranged between 12:1 and 15:1. There was some complication in 29 procedures (53.9\%; Table 3), however they were serious only in 4 patients, leading to TL interruption, and no deaths occurred. Those complications were: respiratory arrest/failure in 2 cases with the need for ventilator support, but in the context of severe pulmonary clinic, arterial hypotension, and mucocutaneous haemorrhage from the puncture sites. The imputability of these serious adverse reactions cannot be attributed to the procedure as they may be due to multiple causes. Hypocalcaemia-related side effects were observed in 22 procedures, but promptly reverted with calcium gluconate administration. Hyperthermia was recorded in one procedure and was reversed with antipyretic. Tachycardia was detected in a patient at the end of the procedure and obstruction of an apheresis kit was detected in another patient, both already at the end of the procedures, which led to their interruption. Note that intravenous calcium was administrated during the procedure on demand, but administering it prophylactically before the procedure was discussed, since a large number of the patients suffer symptoms of hypocalcaemia during the different types of apheresis, therefore this practice is under review. The median haemoglobin pre-TL was $8.3 \mathrm{~g} / \mathrm{dL}(5.2-13.4)$ and post-TL was $8.1 \mathrm{~g} / \mathrm{dL}(5.6-12.2)$. There was a reduction in platelets from a median of $71,000 / \mathrm{mm}^{3}(17,000-379,000)$ to $65,000 / \mathrm{mm}^{3}(16,000-423,000)$. The patients were transfused with RBC concentrates in 21 procedures, platelet transfusions were necessary in 24 , and fresh frozen plasma was required in 16 cases for changes in the coagulation assay. The tubing set of the apheresis equipment was primed with a saline solution. However, in 15 procedures it was decided to use $\mathrm{RBC}$ concentrate corresponding to 7 paediatric patients: 5 of them weighed less 
Table 4. Laboratorial and clinical efficacy and overall survival

\begin{tabular}{|c|c|c|c|c|}
\hline & & \multirow[t]{2}{*}{ Months } & \multicolumn{2}{|c|}{ Overall survival } \\
\hline & & & $\%$ & $95 \% \mathrm{Cl}$ \\
\hline \multirow[t]{2}{*}{ All patients } & & 1 & 64.5 & $49.7-83.8$ \\
\hline & & 6 & 51.6 & $36.7-72.6$ \\
\hline \multirow[t]{4}{*}{ Leukostasis } & None/neurological/pulmonary & 1 & 73.7 & $56.3-96.4$ \\
\hline & & 6 & 63.2 & $44.8-89.0$ \\
\hline & Neurological and pulmonary & 1 & 50.0 & $28.4-88.0$ \\
\hline & & 6 & 33.3 & $15.0-74.2$ \\
\hline \multirow[t]{4}{*}{ Laboratorial efficacy } & With WBC count reduction & 1 & 68.8 & $49.4-95.7$ \\
\hline & & 6 & 56.2 & $36.5-86.7$ \\
\hline & No WBC count reduction & 1 & 60.0 & $39.7-90.7$ \\
\hline & & 6 & 46.7 & $27.2-80.2$ \\
\hline \multirow[t]{4}{*}{ Clinical efficacy } & Improvement & 1 & 93.8 & $82.6-100.0$ \\
\hline & & 6 & 81.2 & $64.2-100.0$ \\
\hline & Same or worse clinical condition & 1 & 33.3 & $16.3-68.2$ \\
\hline & & 6 & 20.0 & $7.3-55.0$ \\
\hline
\end{tabular}

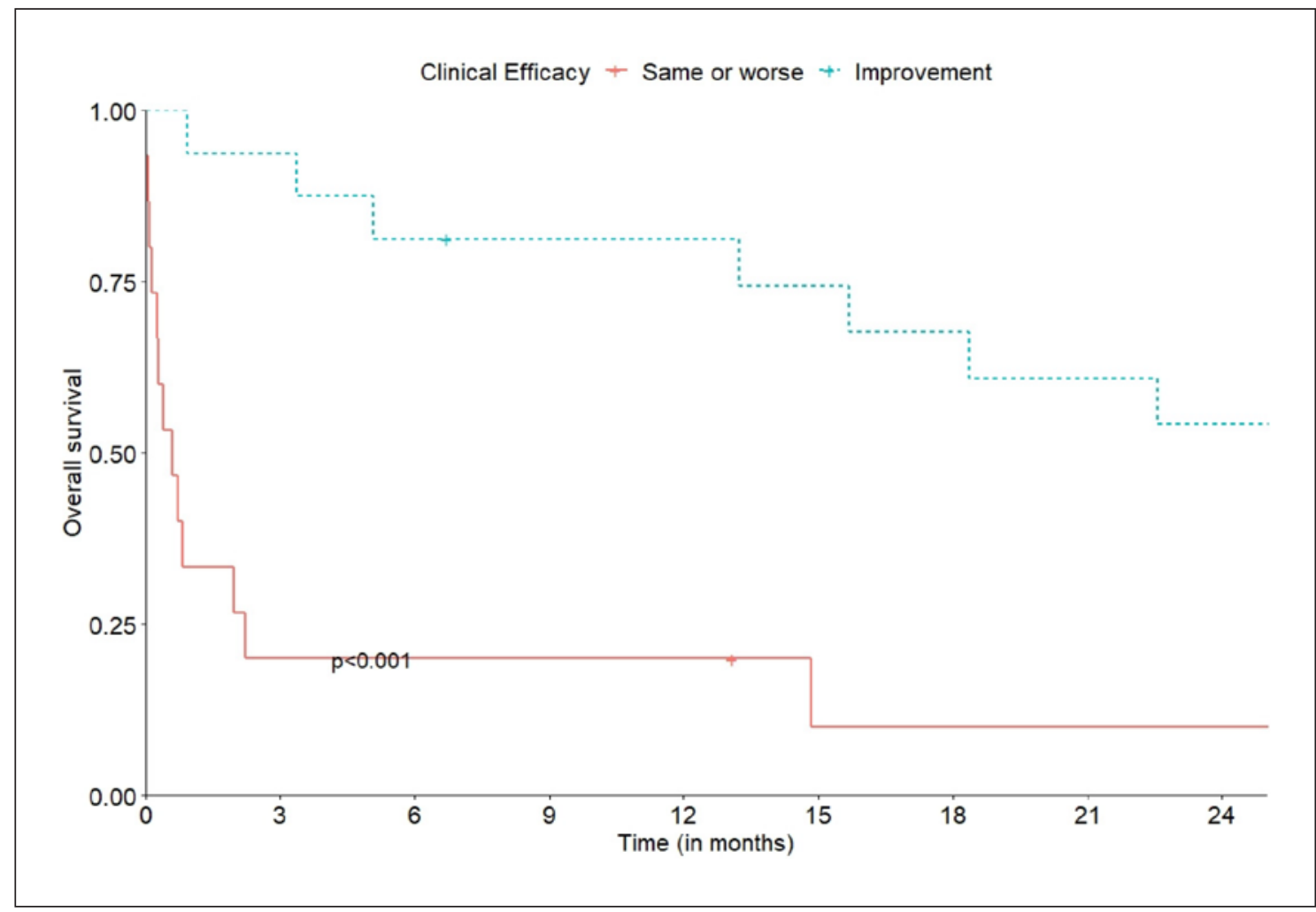

Fig. 1 A significant difference in survival was shown between the group that improved and the group that maintained the same clinical condition or worsened.

than $30 \mathrm{~kg}$, an 8-year-old child weighed $35 \mathrm{~kg}$ with a haematocrit of $21.4 \%$, and another 10 -year-old child weighed $40 \mathrm{~kg}$ and also had a low haematocrit of $23 \%$. After TL sessions, clinical improvement was observed in 16 patients, who survived at least for 1 month. The remaining 15 patients maintained or worsened their condition, and 10 of them had an early death ( $<30$ days). There is a significant difference in survival $(p<0.001)$ between the group that improved and the group that maintained the same clinical condition or worsened (Fig. 1). Note that patients who had clinical improvement had an $81.2 \%$ survival (95\% CI 64.2-100.0) at 6 months, while those who maintained the same condition or worsened had a $20.0 \%$ survival (95\% CI 7.3-55.0) at 6 months (Table 4). In our series of patients who had both neurological and pulmonary manifestations, this was not associated with an in- 


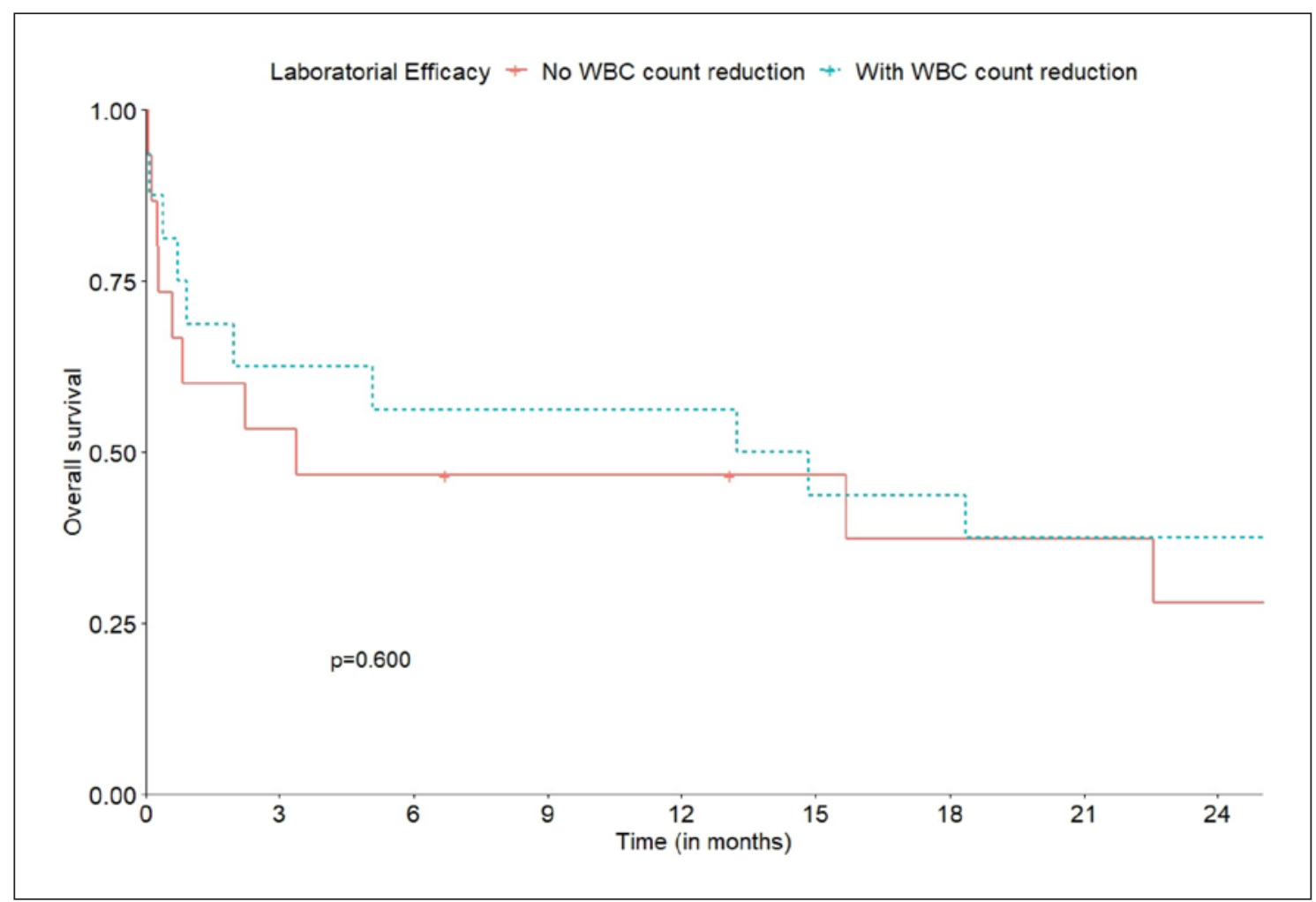

Fig. 2 There was no significant difference in survival between the two groups with and without WBC count reduction.

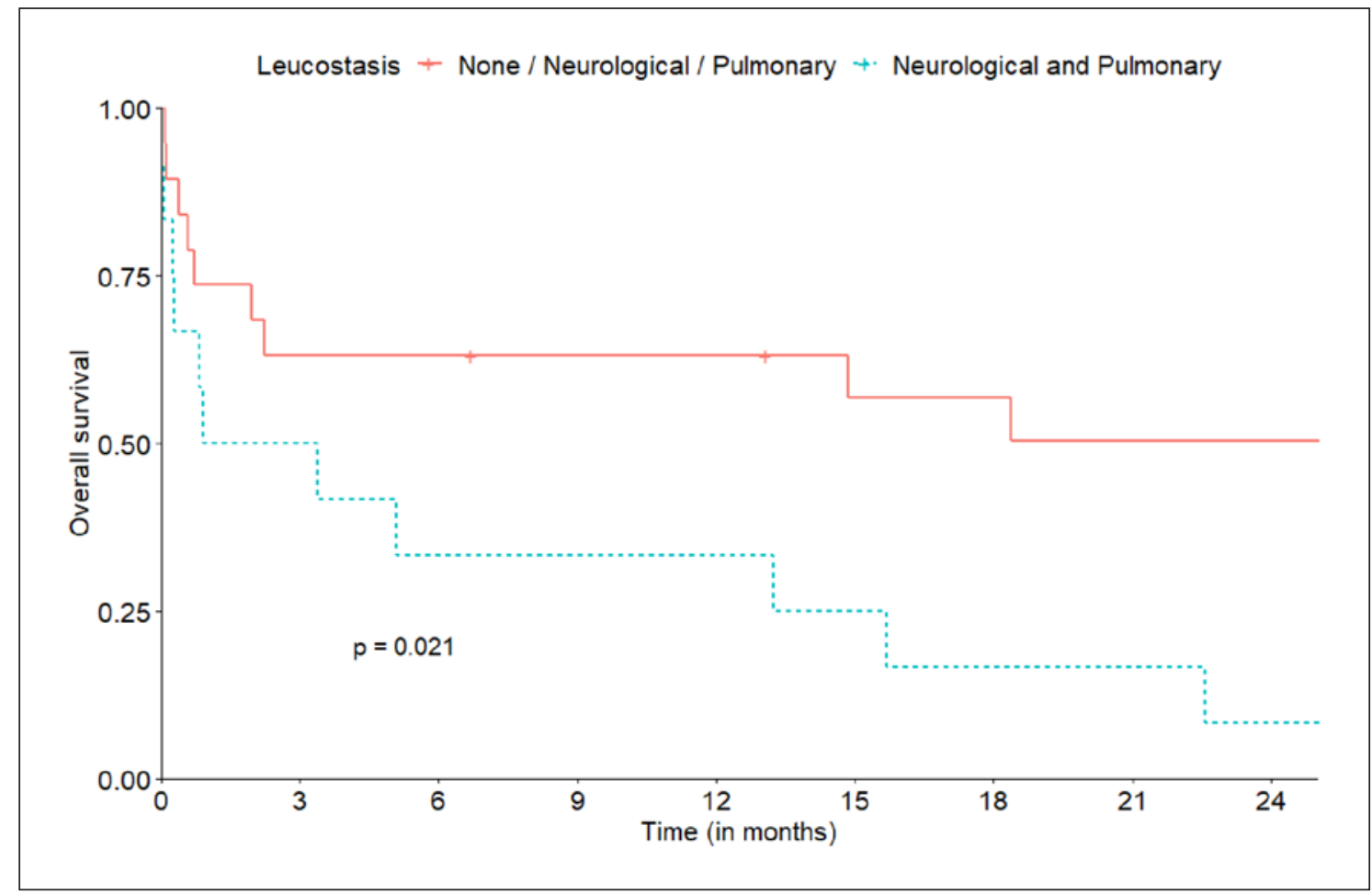

Fig. 3 There was no significant difference in the survival of the group of patients with signs of leukostasis and those without them. 
creased rate of mortality ( $p=0.600)$. Laboratory efficacy after TL occurred in 16 patients, who had a reduction in WBC count, and did not succeed in 15 patients, who had no WBC count reduction. However, in this case there was no significant difference in survival $(p=0.600)$ between the two groups (Fig. 2). The overall survival rate at 6 months after TL was $51.6 \%$ (95\% CI 36.7-72.6) and there was also no significant difference in the survival $(p=$ 0.021 ) of the group of patients with signs of leukostasis and those without them (Fig. 3). The lack of immediate clinical improvement was a sign of a poor prognosis. Regarding patient follow-up, currently 11 of them are alive: 3 patients with ALL diagnosed in paediatric age who are under medical surveillance after chemotherapy at their health centre, 2 patients with ALL as well as 1 patient with AML diagnosed in paediatric age, and 1 AML patient diagnosed in adulthood under medical surveillance in the bone marrow transplantation unit of our centre. Finally, 1 patient with AML relapsed in treatment, and 3 with CML in the chronic phase under protein kinase inhibitors, followed up at the onco-haematology service of our hospital. The causes of death in the remaining 20 patients were mainly acute respiratory distress syndrome, sepsis, and the progression of the underlying disease.

\section{Discussion}

In our centre, most patients who underwent TL were critically ill, but despite this the survival rate was similar to that reported in the literature. The overall survival rate at 6 months after TL was 51.6\%. In terms of diagnoses, clinical presentation of leukostasis, and causes of death, the characteristics of our case series are also similar to those of other published studies (51.6\% of patients were diagnosed with AML, $83.9 \%$ of patients had leukostasis, and $65 \%$ of patients died of ARDS and/or sepsis). Conclusions were limited due to the reduced number of patients in the study, but despite the small size and limited homogeneity of our cases, we can conclude that TL is a safe and efficacious therapeutic measure for leukoreduction in haematological pathologies. Mild complications related to TL were very likely to occur in $44.4 \%$ of sessions (paraesthesia, bleeding from the puncture sites, and apheresis kit obstruction). A $39.1 \% \mathrm{WBC}$ reduction was obtained and there were serious complications only in 4 patients, but without attributing causality, so it may have represented a slight improvement in leukostasis management in these patients. The EI revealed a reliable and easily calculated indicator and would be important to find standard indicators to technically and clinically monitor the $\mathrm{TL}$ in order to allow multicentre comparisons from the data available. In this sense, an international registry of patients treated with TL could be carried out by special- ists, included in an existing apheresis association, or in a register created by a haematology society with the aim of having bases to guide different situations in a more supported way. TL is being used less and less because the treatments for haematological diseases have undergone a great evolution; however, it remains an option in a restricted group of cases, mainly to reduce complications associated with HL until systemic intensive chemotherapy commences if the risk does not contraindicate it.

\section{Acknowledgements}

The authors are grateful to the different nurses and medical staff who participated in the leukapheresis procedures during this period and are also grateful to Jessica Rodrigues, from the Cancer Epidemiology Group, who analysed the data that allowed us to draw conclusions.

\section{Statement of Ethics}

The hospital ethics committee authorised this review with the data collection necessary for its performance. Confidentiality and anonymity was guaranteed.

\section{Conflict of Interest Statement}

The authors declare no competing financial interests and no conflicts of interest regarding this article.

\section{Funding Sources}

The retrospective analysis of the clinical files of the patients was carried out by the authors without sponsors or funding. For the article processing charge, we received support from the company Terumo BCT.

\section{Author Contributions}

All authors made a substantial contribution to the manuscript and declare that they agree to the rules to publish the article.

\section{Data Availability Statement}

The authors consulted the clinical files and analysed the intended data. All data generated or analysed during this study are included in this article and its supplementary material files. Further enquires can be directed to the corresponding author. 


\section{References}

1 Aqui N, O'Doherty U. Leukocytapheresis for the treatment of hyperleukocytosis secondary to acute leukemia. Hematology Am Soc Hematol Educ Program. 2014;2014:457-60.

2 Ganzel C, Becker J, Mintz PD, Lazarus HM, Rowe JM. Hiperleukocytosis, leukostasis and leukapheresis: practice management. Blood Rev. 2012;26:117-22.

3 Bewersdorf JP, Giri S, Tallman MS, Zeidan AM, Stahl M. Leukapheresis for the management of hyperleukocytosis in acute myeloid leukemia: a systematic review and meta-analysis. Transfusion. 2020;60:2360-9.

4 Connelly-Smith LS, Linenberger ML. Therapeutic apheresis for patients with cancer. Cancer Control. 2015;22(1):60-78.

5 Rolling C, Ehninger G. How I treat hyperleukocytosis in acute myeloid leukemia. Blood. 21 May 2015;125(21):3246-52.

6 Coffe C, Pouthier F, Barisien C, Slimane M, Sheytanova A. Therapeutic leukapheresis and thrombapheresis in medical emergencies. Transfus Apher Sci. 2020;59:102997.

7 Nath RM, Kakkar B, Chowdhry M, Agrawal S, Seth S, Thakur UK. Therapeutic leukapheresis in a tertiary care hospital: a case series. Asian J Transfus Sci. 2017;11(1):65-8.

8 Villgran V, Agha M, Raptis A, Hou JZ, Farah $\mathrm{R}$, Lim SH, et al. Leukapheresis in patients newly diagnosed with acute myeloid leukemia. Transfus Apher Sci. 2016;55:216-20.

9 Tan D, Hwang W, Goh YT. Therapeutic leukapheresis in hyperleukocytic leukaemias - the experience of a tertiary institution in Singapore. Ann Acad Med Singap. 2005;34(3):229-34.

10 Korkmaz S. The management of hyperleukocytosis in 2017: do we still need leukapheresis? Transfus Apher Sci. 2018;57:4-7.

11 Howell C, Douglas K, Cho G, El-Ghariani K, Taylor P, Potok D, et al. Guideline on the clinical use of apheresis procedures for the treatment of patients and collection of cellular therapy products. British Committee for Standards in Haematology. Transfus Med. 2015;25:57-78.

12 Padmanabhan A, Connelly-Smith L, Aqui N Balogun RA, Klingel R, Meyer E, et al. Guidelines on the use of therapeutic apheresis in clinical practice - evidence-based approach from the writing committee of the american society for apheresis: the eighth special issue. J Clin Apher. 2019;34:171-354.

13 Blum W, Porcu P. Therapeutic apheresis in hyperleukocytosis and hyperviscosity syndrome. Semin Thromb Hemost. 2007;33(4): 350-4.

14 IngridParra MS, Victoria P, Rodriguez G, José A, García-Erce . Therapeutic leukapheresis: 9-year experience in a university hospital. Blood Transfus. 2015;13:46-52.

15 Stenzinger M, Bonig H. Risks of leukapheresis and how to manage them: a non-systematic review. Transfus Apher Sci. 2018;57:5628-634. 